\title{
ROAD EXTRACTION FROM HIGH RESOLUTION REMOTE SENSING IMAGE USING MULTIRESOLUTION IN CASE OF MAJOR DISASTER
}

\author{
Idrissa Coulibaly, N. Spiric, M. Ouled Sghaier, W. Manzo-Vargas, R. Lepage, M. St-Jacques \\ École de technologie supérieure, Montréal, Québec, Canada \\ E-mail : icoulibaly@livia.etsmtl.ca
}

\begin{abstract}
Road extraction is a topical research because of complexity due to his large topological variability. Increasing the spatial resolution generates noise which makes extraction difficult, especially in case of major disaster in an urban context. This problem increases false alarm rates and generally affects the performance of road extraction algorithm. Our aim is to improve the quality of roads extraction after adaptation of the Lowe's SIFT descriptors (scale-invariant feature transform) jointly with spectral angle algorithm. The characterization is performed on two image at various resolution images, respectively representing a rural and urban disaster area, captured by Quickbird satellite. Our approach significantly reduces the amount of false detection and shows an overall accuracy of up to nearly $30 \%$ in some cases.
\end{abstract}

Index Terms - Road extraction, Multiresolution analysis, Multispectral images, Major disaster, Spectral angle.

\section{INTRODUCTION}

In recent years, occured a growing number of natural disasters around the world: the tsunami in Indian Ocean (2004), the earthquake in Haiti (2010) and the earthquake in Japan (2011) are recent examples [1]. All relevant scenes are spread over a wide area and in all cases the damage was immense.

One prerequisite for good care organization of assistance is the availability of reliable and quick information about data on the nature of the operational or damaged networks (communication networks, networks land transport, energy and hydroelectric networks). The mapping of those infrastructures and their respective status remains a major turning point for first responders and experts from international organizations just after the disaster. This is a request constantly made upon activation of the International Charter on Major Disasters [2], [3].

The use of remote sensing imagery to accomplish this task is one of the best options, because of the fast availability of the image data and the wide coverage of the affected areas [3]. The precision of the obtained image and its high spatial resolution are undeniable contributions. But the high accuracy of the resolution generates a cost: the increase of the volume of information to be processed and the level of detail that generates noise. Indeed, the details disturb the detection of the roads by the presence of artifacts and visible objects such as cars, shrubs and buildings. This problem increases the false alarm rate and affects the performance of road detection and extraction algorithms. Nowadays, the information provided by the image is manually extracted by photointerpreters. This process is hard and the analysis time delay can have consequences for victim's life. So we need to automate or assisting photointerpreters during the map production showing the state and road network conditions in a reasonable time. In this study, we propose to improve the quality of automatic detection of the roads from Quickbird optical images with very high spatial resolution in order to extract roads with a significant reduction of false alarms. In an image, a large variability of the road characteristics and some details that characterize these linear structures are observed. Those structures change according to the image resolution, we had developed a new multi-scale method. This new approach is an adaptation of the Lowe's SIFT descriptors [4]. It helps to highlight relevant information without the need for additional processing, and to remove details that are not required for subsequent analysis [5]. To appreciate the detectability degree of road graphs, we use the algorithm for extracting roads based on the spectral angle [6]. To objectively measure the evolution of the detection results, we considered three metrics: recovery rate, accuracy rate and quality [7], [8]. Our approach present a reduction in false alarms and a significant gain in the quality of detection. Our paper is organized as follows: In Sect. 2 , we introduce pyramid model representation based on the scale-space behavior of roads and spectral angle and its implementation. The Sect. 3, we present our flowchart and the different steps included. While the Sect. 4 shows that the approach gives reasonable results for which we have evaluated the performance based on a reference image. We conclude the paper with the summary in Sect. 5.

\section{PYRAMID MODEL AND IMPLEMENTATION}

The appearance of roads in a remote sensing image depends on the spectral sensibility [9] as well as on the resolution of

(C) 2014 IEEE. Personal use of this material is permitted. Permission from IEEE must be obtained for all other uses, in any current or future media, including reprinting/republishing this material for advertising or promotional purposes, creating new collective works, for resale or redistribution to servers or lists, or reuse of any copyrighted component of this work in other works.

The final publication is available at http://dx.doi.org/10.1109/IGARSS.2014.6947035 
the sensor. This scale depends on the resolution of the image. Notably, a road can appear in different ways depending on the scale of observation. One way to describe an image and its components resides in generating simple versions of the image and analyse the level of information lost following successive simplifications of the image. The multiresolution analysis (MRA) was first introduced by [10]. The general principle of the algorithm, described in figure 1, can be summarized in two successive operations applied on an image: the low-pass filtering with a Gaussian kernel and the subsampling. The advantage of using a Gaussian kernel has been demonstrated in [11] to be potentially the only one to require characteristic properties for multi-scales representation.

The pyramidal approaches are mostly performed jointly with semi-automatics road extraction methods, due to the difficulty in selecting a proper scale level for an object recognition application. According to [12], the authors suggest a technique based on a multi-scale processing combined to a technique of detection by active contours also known as snakes. On the other hand, according to [13], the authors discuss the case of automatic road detection on an optical image with high spatial resolution in urban area, using a step of multi-scale pre-processing. The main encountered problem is the invariance of scale face to refined operations or to evaluate image descriptors [11]. The SIFT algorithm of Lowe [4] allows a performance improvement of objects recognition that is invariant to the scale. The pyramidal representation is based on the detection step of the SIFT key points algorithm. The method uses a concept of representation with multiple trapezoidal pyramids, which allows processing of multispectral images at various resolution levels and scales. The treatment is performed on three dimensions: the Cartesian coordinates $(x, y)$ and the scale factor $\sigma$. The spatial scales extremes need to be identified with Difference-of-Gaussian (DoG) between two images of adjacent scales. This technique of DoG is equivalent to that we used for our tests. The images are distinguished by factors of scales separated by a fixed coefficient $(k)$, calculated to discretize the space of scales following a geometrical progression $\sigma, k \sigma, k^{2} \sigma, \ldots, 2 \sigma$. The decomposition allows preserving the invariance of scales for the local extrema detection at different scales.

\section{METHODOLOGY}

\subsection{Generation of scale-space}

Our overall pattern is divided into three blocks (figure 2). The first block generates a serie of scaled images with the same level of resolution by a progressive Gaussian filtering of each band horizontally. It is necessary to decompose the multispectral image by individual bands for filtering discretly (figure 3).

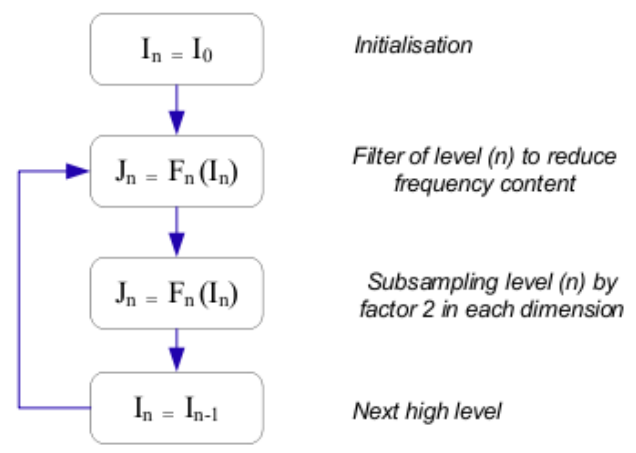

Fig. 1. Process for building a pyramid with (n) levels

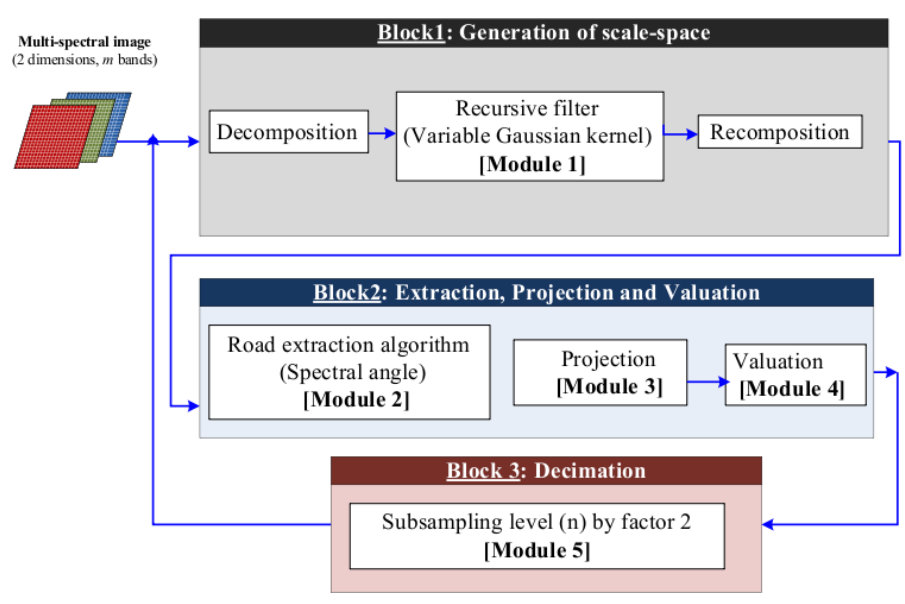

Fig. 2. The flowchart showing different steps and corresponding sections of the proposed method

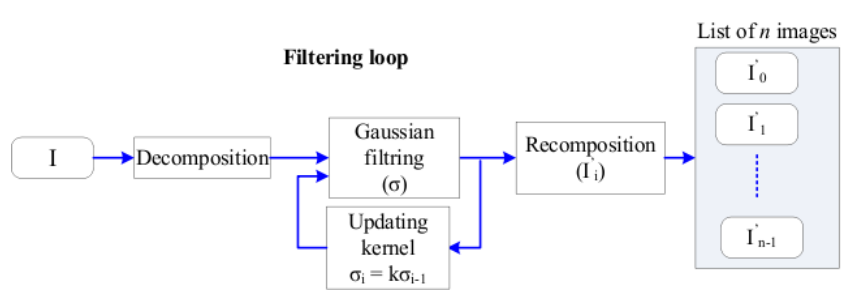

Fig. 3. Block1 [Module 1]: Recursive filtering loop.

\subsection{Road extraction, selective projection and valuation}

The second block consists in the application of the roads extraction algorithm on each reconstituted images. The results of this detection are projected to the original image for comparison purposes.

In [6], the authors present an interesting road extraction technique. The technique uses the spectral information to distinguish roads to other land uses. For a bands multi-spectral image, the spectral angle is taken between the reference vector pixel and the current vector pixel. For a multispectral im- 
age with $M$ spectral bands, the spectral angle is defined as:

$$
A S=\cos ^{-1}\left(\sum_{b=1}^{M} r(b) \cdot p(b) / \sqrt{\left.\sum_{b=1}^{M} r(b)^{2} \sum_{b=1}^{M} p(b)^{2}\right)}\right.
$$

$b$ being the spectral band, $r$ is the reference pixel and $p$ the current pixel.

The smaller the angle, the greater the similarity between the evaluated pixel and the reference. The resulting image contains all roads in a darker color. We apply to the image of this spectral angle a line detection algorithm that uses a gradient constraint to determine the pixels delineating the road. Removed of non maximum scalar values and the highest scalar value is obtained between $a_{1}$ and $b_{1}$ (figure 4 ). This method is described in [14]. A quantitative assessment is

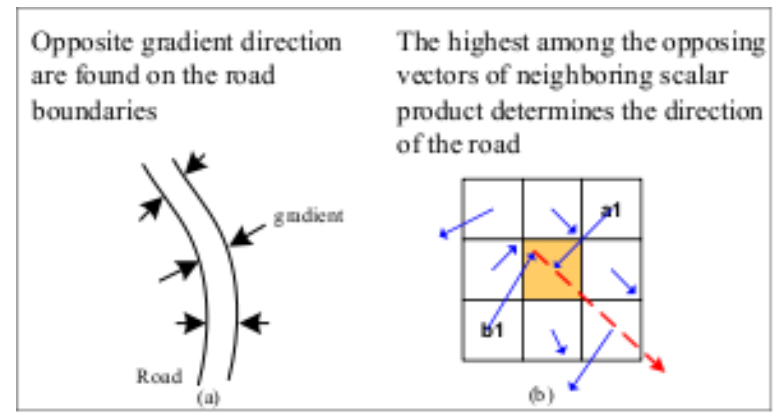

Fig. 4. Block2 [Module2], gradient representation and the roads: (a) gradient direction toward the roads; (b) computation of the direction and scalar values for each pixel.

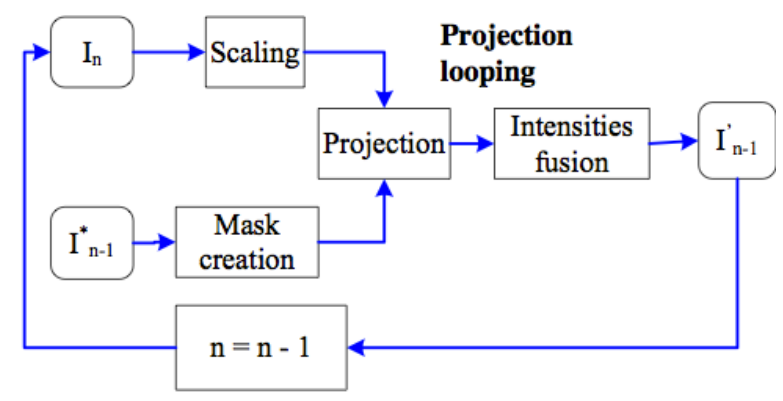

Fig. 5. Block2 [Module3]: Projection looping.

performed to measure the degree of precision $(\mathrm{P})$, recovery $(\mathrm{R})$ and quality $(\mathrm{Q})$ of the obtained results.

\subsection{Decimation}

Finally, the third block is devoted to reducing the resolution (subsampling by factor 2) in preparation for the second level of pyramid processing. These operations are performed in a loop for four octaves with four images by scale level, a total of 16 images generated for each series of tests.

\section{RESULTS AND INTERPRETATIONS}

All the results are obtained within the Orfeo Toolbox (OTB) framework ${ }^{1}$. The OTB is an open source set of tools for remote sensing data exploitation. Rural and disasters images (Haiti) from the Quickbird satellite, representing increasing levels of complexity in terms of road density and interconnections were used for testing. For the results assessment, only main roads were considered. In figures 7 and 8 , a relative decrease in false alarms at residential area was observed. A significant reduction explains a high recovery rate for the urban image. The application of MRA, increased the recovery rate over $96 \%$, with a relative decrease in precision, about $20 \%$ in some cases. The presence of false alarms affecting the recovery rate is partly due to the choice of the reference pixel. The quality of extraction is increased by over $30 \%$ in average. Any failures can be justified by the degree of spreading of the distributions concerning each band radiometric information, the application contexts, the road topology and the kernel parameter.

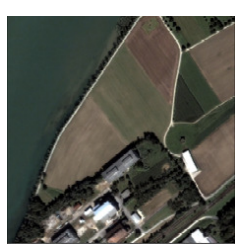

(a) Rural image

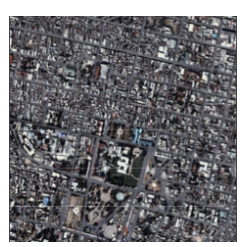

(d) Haiti image

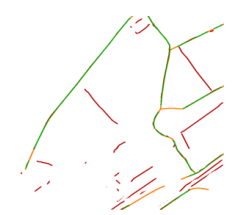

(b) Extracted road

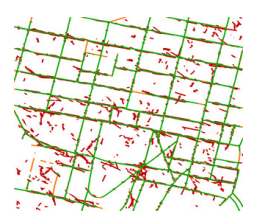

(e) Extracted road

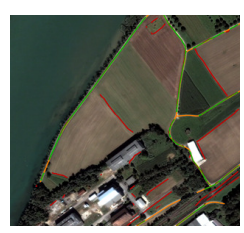

(c) Overlay

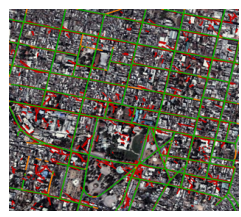

(f) Overlay
Fig. 6. Road extraction results on two images with our multiresolution algorithm. Legend: [Green=good matching]; [Red=incorrect matching]; [Orange $=$ Requires verification]

\section{CONCLUSIONS AND FUTURE WORK}

The experimental results are very encouraging and show that the MRA can significantlyimproves segmentation of objects under certain conditions. The application of our approach increases the recovery rate for about $5 \%$ with a gain of $30 \%$ of roads detection quality. The main advantage of this technique is that it eliminates iteratively the problem of the noise present in the images. To improve the recovery rate we could consider the average width of the roads and the context (rural or urban) of the image in the modelling method. The choice of the reference pixel based solely on spectral information instead of

\footnotetext{
${ }^{1}$ The ORFEO toolbox software guide. http://otb.cnes.fr, 2007
} 


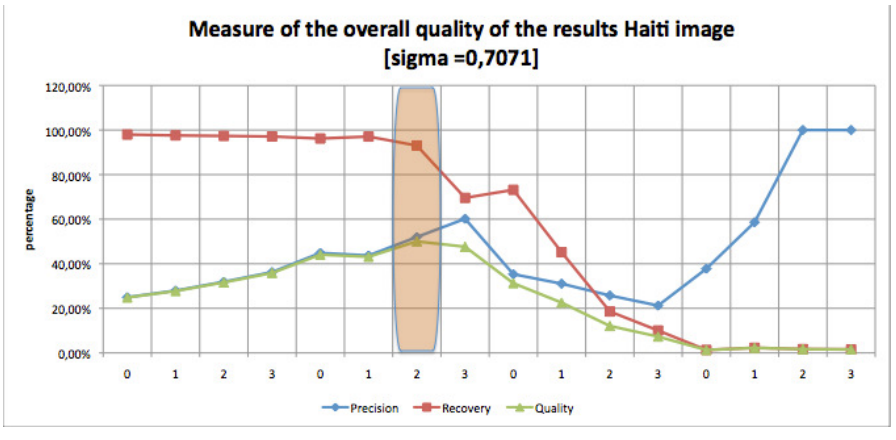

Fig. 7. development of metrics for Haiti image

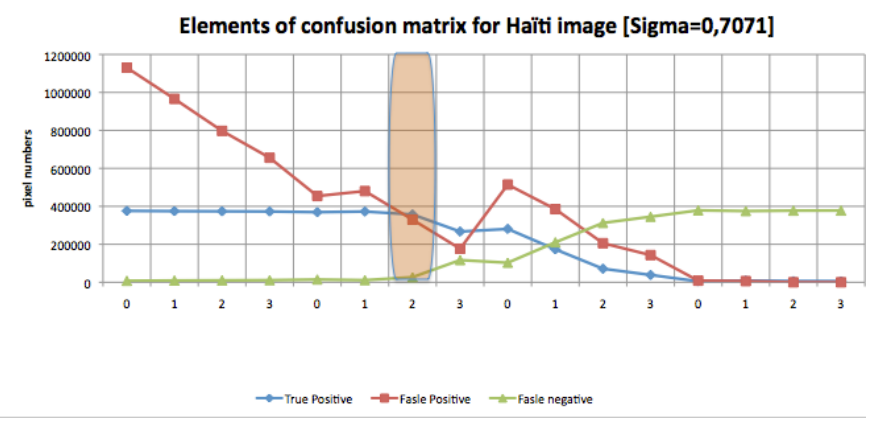

Fig. 8. Number of pixels per class for the Haiti image

the texture could bring an added value and significantly reduce false alarms. Results could be improved by exploring tracking algorithms such as active contours. To improve detection rate and reduce false alarms, we must explore other avenues such as adding spatial information (texture), tracking algorithms roads (active contours-Snakes, Kalman filter, etc.).

\section{REFERENCES}

[1] J. L. Bessis, J. Bquignon, and A. Mahmood, "The international charter" space and major disasters " initiative," Acta Astronautica, vol. 54, no. 3, pp. 183-190, 2004.

[2] "International charter space and major disasters: Emergency on-call officer procedure, charte internationale scenario guidelines, available online at: http://www.disasterscharter.org," Tech. Rep., Charte Internationale Scenario Guidelines, 2008.

[3] J. Inglada and A. Giros, "On the real capabilities of remote sensing for disaster management - feedback from real cases," IGARSS '04: Geoscience and Remote Sensing Symposium, vol. 2, pp. 1110-1112, 2004.

[4] David G. Lowe, "Distinctive image features from scaleinvariant keypoints," International Journal of Computer Vision, vol. 60, no. 2, pp. 91-110, 2004.
[5] S. Hinz and A. Baumgartner, "Automatic extraction of urban road networks from multi-view aerial imagery," ISPRS Journal of Photogrammetry and Remote Sensing, vol. 58, no. 1, pp. 83-98, 2003.

[6] E. Christophe and J. Inglada, "Robust road extraction for high resolution satellite images," IEEE International Conference on Image Processing, 2007. ICIP 2007, vol. 5, pp. 437-440, 2007.

[7] J. Senthilnath, M. Rajeshwari, and S. Omkar, "Automatic road extraction using high resolution satellite image based on texture progressive analysis and normalized cut method," Journal of the Indian Society of Remote Sensing, vol. 37, no. 3, pp. 351-361, 2009.

[8] U. Bacher and H. Mayer, "Automatic road extraction from multispectral high resolution satellite images," Proceedings of CMRT05, 2005.

[9] R. Tonjes and S. Growe, "Knowledge based road extraction from multisenor imagery," International Archives of Photogrammetry and Remote Sensing, vol. 32, pp. 387-393, 1998.

[10] S.G. Mallat, "A theory for multiresolution signal decomposition: The wavelet representation," IEEE Transactions on Pattern Analysis and Machine Intelligence, vol. 11, no. 7, pp. 674-693, 1989.

[11] T. Lindeberg, "Scale-space theory: A basic tool for analyzing structures at different scales," Journal of applied statistics, vol. 21, no. 1-2, pp. 225-270, 1994.

[12] I. Laptev, H. Mayer, T. Lindeberg, W. Eckstein, C. Steger, and A. Baumgartner, "Automatic extraction of roads from aerial images based on scale space and snakes," Machine Vision and Applications, vol. 12, no. 1, pp. 2331, 2000.

[13] H. Long and Z. Zhao, "Urban road extraction from highresolution optical satellite images," International Journal of Remote Sensing, vol. 26, no. 22, pp. 4907-4921, 2005.

[14] V. Lacroix and M. Acheroy, "Feature extraction using the constrained gradient," ISPRS Journal of Photogrammetry and Remote Sensing, vol. 53, no. 2, pp. 85-94, 1998. 